\title{
Efficacy of prolonged tapered and pulsed vancomycin regimen on recurrent Clostridioides difficile infection in the Japanese setting: a case control study
}

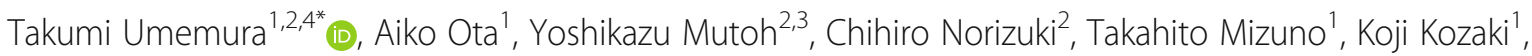
Yoshiaki Ikeda ${ }^{4}$ and Toshihiko Ichihara ${ }^{2}$

\begin{abstract}
Background: According to the Clinical Practice Guidelines for Clostridioides difficile, oral vancomycin is to be used in vancomycin tapered and pulsed regimen (VCM-TP) for recurrent Clostridium difficile infection (CDI). However, data on the efficacy of VCM-TP in Japanese patients with recurrent CDI are scarce. To address this gap, we investigated the efficacy of VCM-TP and performed a case-controlled study to assess the risk factors associated with treatment failure in these patients.

Findings: We conducted this study on all patients who were administered VCM-TP for recurrent episodes of CDI between January 2008 and December 2018 at Tosei General Hospital. All patients had documented follow-ups within 90 days after completion of the VCM-TP. Data were obtained for comparative analysis of treatment success or failure. Thirty-six patients were eligible for this study, and treatment success was documented in 23 patients (63.9\%) following VCM-TP treatment. Treatment success was documented in 22 of 30 (73.3\%) patients who received the recommended therapy according to the Clinical Practice Guidelines. The frequency of patients treated with the recommended therapy was higher in the treatment success group (95.7\%) than in the treatment failure group (61.5\%) (OR: 13.75, 95\% Cl: 1.39-136.39, $p=0.016)$. Vancomycin-resistant enterococci culture tests were performed in 20 patients (55.6\%), and all results were negative.

Conclusions: Our findings suggest that VCM-TP is a good therapeutic option for recurrent CDI in Japanese patients. Furthermore, administration of the recommended VCM-TP is important for achieving a high rate of treatment success. Hence, antimicrobial stewardship teams should support the implementation of recommended VCM-TPs.
\end{abstract}

Keywords: Vancomycin, Vancomycin tapered and pulsed dose regimen, Clostridioides difficile infection

\section{Background}

Clostridioides difficile infection (CDI) is one of the nosocomial infections associated with poor outcome $[1,2]$ and involves a huge medical expense [3, 4]. The 30-day mortality rate associated with CDI is $8 \%-31 \%$ [5], and the rate of recurrence is $16 \%-24 \%$ despite successful treatment of the initial episode [6]. After the first

\footnotetext{
* Correspondence: umemuratakumi@gmail.com

'Department of Pharmacy, Tosei General Hospital, 160, Nishi oiwakecho, Seto, Aichi 489-8642, Japan

${ }^{2}$ Department of Infection and Prevention, Tosei General Hospital, 160, Nishi oiwakecho, Seto, Aichi 489-8642, Japan

Full list of author information is available at the end of the article
}

episode of recurrence, the rate of second CDI recurrence is $22.6 \%-41.8$ [7], and the medical expenditure in patients with recurrent CDI is higher than that in patients with non-recurrent CDI [8]. According to the Clinical Practice Guidelines for Clostridium difficile by the Infectious Diseases Society of America (IDSA) and the Society for Healthcare Epidemiology of America (SHEA), oral vancomycin should be used in a vancomycin tapered and pulsed regimen (VCM-TP) for a standard 10-day course to treat the initial episode of CDI [9]. The Clinical Practice Guidelines by the Japanese Society of Chemotherapy and The Japanese Association for Infectious Diseases recommend using VCM-

(c) The Author(s). 2019 Open Access This article is distributed under the terms of the Creative Commons Attribution 4.0 International License (http://creativecommons.org/licenses/by/4.0/), which permits unrestricted use, distribution, and 
TP for the first or for $\geq 2$ recurrence of CDI as an alternative therapy [10]. Despite reports of the efficacy of VCM-TP for recurrent CDI $[11,12]$, data on Japanese patients with recurrent CDI remain scarce. Therefore, we investigated the efficacy of VCM-TP in Japanese patients with recurrent CDI and performed a case-controlled study to assess the risk factors associated with treatment failure from recurrent CDI.

\section{Methods}

\section{Study samples}

This study was approved by the ethics committee of the Tosei General Hospital (receipt No. 769). This study was conducted on all patients who were administered VCMTP for recurrent episodes of CDI (defined as symptoms of re-infection within 8 weeks of the prior episode) between January 2008 and December 2018 at Tosei General Hospital, a 633-bedded hospital. VCM-TP was defined as tapering vancomycin administration frequency in the sequence of once daily (pulse phase), and then every other day or every third day dosing for at least 2 weeks [13]. All patients had at least one of their prior CDI episodes confirmed by a positive test for the $C$. difficile toxin in their stool. For detecting the toxin, lateral flow immunoassay was performed in the stool specimens using Uniquick (Kanto Kagaku Kanto Kagaku Co., Ltd., Tokyo from January 2008 to June 2011), X/pect toxin A/B (Kanto Kagaku Co., Ltd., Tokyo from July 2011 to September 2014) and C. Diff Quik Chek Complete (Alere Medical Co. Ltd., Tokyo from October 2014 to December 2018).

\section{Efficacy of VCM-TP}

All patients were followed up for more than 90 days after completion of VCM-TP. Treatment success was defined as the non-recurrence of diarrheal symptoms that require re-treatment with a CDI-specific agent [13]. Some patients noted a transient recurrence of symptoms near the end or just after completion of the pulse phase of the VCM-TP, and it resolved spontaneously, usually within a day; these episodes were not considered as recurrent CDI [13]. We investigated the treatment success rate in all patients with VCM-TP, in patients following the recommended VCMTP according to the Clinical Practice Guidelines (125 mg four times per day for 10-14 days, two times per day for a week, once per day for a week, and every 2 or 3 days for 2-8 weeks) [9], and in patients following inappropriate VCM-TP therapy, which is defined as VCM-TP that departs from the recommended therapy.

\section{Factors associated with treatment failure in the administration of VCM-TP}

The following data were obtained for comparative analysis of treatment success or failure: age, sex, underlying diseases (hypertension, diabetes mellitus, prior abdominal surgery, gastroesophageal reflux, immunocompromised, chronic kidney disease, chronic liver disease, and malignancy according to Sirbu et al. [13]), number of CDI episodes, duration of treatment, Charlson comorbidity index, drug use (proton pump inhibitors, histamine receptor-2 blockers, probiotics, antidiarrheals, and antibiotic use 90 days prior to the treatment), concomitant antibiotic use with VCM-TP treatment, disease severity (according to the Zar criteria and the MN criteria $[14,15])$, and recommended VCM-TP according to the Clinical Practice Guidelines.

\section{Detection of vancomycin-resistant enterococci during or after VCM-TP}

Stool culture tests for vancomycin-resistant enterococci (VRE) were performed between 14 days after initiation and 90 days after completion of VCM-TP to confirm the presence of VRE. The BD Vancomycin-Resistant Enterococci

Table 1 Characteristics of patients with Clostridioides difficile infection treated with vancomycin tapered and pulsed-dose regimen

\begin{tabular}{|c|c|}
\hline Items & $n=36$ \\
\hline Male sex (\%) & $19(52.8)$ \\
\hline Age, median $\left(\mid \mathrm{QR}^{1}\right)$ & $80.5(21-95)$ \\
\hline No. of CDI episodes, mode (range) & $3(2-7)$ \\
\hline Duration of treatment day, median $\left(\mathrm{IQR}^{1}\right)$ & $43(36.8-55)$ \\
\hline \multicolumn{2}{|l|}{ Underlying disease (\%) } \\
\hline Hypertension & $12(33.3)$ \\
\hline Malignancy & $10(27.8)$ \\
\hline Immunocompromised & $9(25.0)$ \\
\hline Diabetes mellitus & $5(13.9)$ \\
\hline Prior abdominal surgery & $5(13.9)$ \\
\hline Chronic kidney disease & $4(11.1)$ \\
\hline Chronic liver disease & $1(1.5)$ \\
\hline Charlson comorbidity index, median (range) & $2(0-8)$ \\
\hline \multicolumn{2}{|l|}{ Drug use (\%) } \\
\hline Proton pump inhibitors & $16(44.4)$ \\
\hline Histamine receptor-2 blockers & $3(8.3)$ \\
\hline Probiotics & $31(86.1)$ \\
\hline Antidiarrheals & $0(0.0)$ \\
\hline Antibiotic use prior 90 days & $33(91.7)$ \\
\hline \multicolumn{2}{|l|}{ Disease severity (\%) } \\
\hline \multicolumn{2}{|l|}{ Zar criteria [14] } \\
\hline$>2$ & $5(13.9)$ \\
\hline$\leq 2$ & $31(86.1)$ \\
\hline \multicolumn{2}{|l|}{ MN criteria [15] } \\
\hline mild & $13(36.1)$ \\
\hline moderate & 19 (52.8) \\
\hline severe & $3(8.3)$ \\
\hline
\end{tabular}

${ }^{1} \mathrm{IQR}$ (interquartile range) 
Selective Agar (Becton Dickinson@, NJ, USA) was used to detect VRE, and Microscan Walkaway system (Beckman Coulter, California, USA) was used to identify VRE at the species level and to determine antimicrobial susceptibility. All cultures were considered resistant according to the breakpoints defined by the Clinical \& Laboratory Standards Institute (M100 S-22).

\section{Statistical analysis}

The qualitative and stratified continuous variables were compared using Fisher Exact test or Pearson $x^{2}$ test. The continuous variables were compared using Mann-Whitney $\mathrm{U}$ test. The predictive values are presented as the odds ratios (ORs) with respective 95\% confidence intervals (CI). Two-tailed $p<0.05$ indicated statistical significance. All analyses were performed using IBM SPSS Statistics version $25\left(\mathrm{IBM}^{\circ}\right)$.

\section{Results}

\section{Efficacy of VCM-TP}

The study included 38 consecutive patients treated with VCM-TP for recurrent CDI. Two patients who died from unrelated disease before completing their regimens were excluded from the analysis. Table 1 shows the characteristics of the included patients. Among the remaining $36 \mathrm{pa}-$ tients, the median number of CDI episodes was 3 (range, 2-7). Twenty-nine patients were male, and the median age was 80.5 years. The median duration of treatment was 43 (IQR, 36.8-55) days. Treatment success occurred in 23 of 36 (63.9\%) patients following VCM-TP and in 22 of 30 patients $(73.3 \%)$ following the recommended therapy. On the contrary, in patients with inappropriate therapy, treatment success occurred in 1 of 6 patients (16.7\%), which is less than the success rate of those following the recommended therapy $(p=0.016)$ (Fig. 1).
Factors associated with treatment failure after administration of VCM-TP

Table 2 shows the comparative characteristics of patients in the treatment success and failure groups. The proportion of patients treated with the recommended therapy was higher in the treatment success group than in the treatment failure group (OR: $13.75,95 \%$ CI: $1.39-136.39, p=0.016$ ). No statistically significant difference was observed between the two groups. The reasons for inappropriate use of VCM-TP in treatment failure patients were the inability of the patients to take vancomycin two times a day for a week (3 cases) and unnecessary washout period during treatment ( 2 cases).

\section{Detection of VRE during or after VCM-TP}

VRE culture was performed in 20 patients (55.6\%) after $41.9 \pm 17.7$ (mean \pm standard deviation) days of VCM-TP treatment initiation, and all were negative.

\section{Discussion}

VCM-TP is recommended for the treatment of recurrent CDI, but a few studies have evaluated the efficacy of VCMTP in Japanese patients with recurrent CDI. Therefore, we investigated the efficacy of VCM-TP in Japanese patients with recurrent $\mathrm{CDI}$ and performed a case-controlled study to assess the risk factors associated with treatment failure from recurrent CDI.

VCM-TP for recurrent CDI resulted in clinical improvement in $63.9 \%$ of all patients, and $73.3 \%$ of patients under the recommended therapy had clinical improvements (Fig. 1). McFarland et al. and Sirbu et al. reported the cure rate of recurrent CDI with VCM-TP as 69.0\% (20/29 cases) and $74.0 \%$ (74/100 cases), respectively [11, 13]. Therefore, the effects of the recommended VCM-TP therapy in Japanese patients are similar to its effects in non-Japanese patients. Furthermore, our results showed

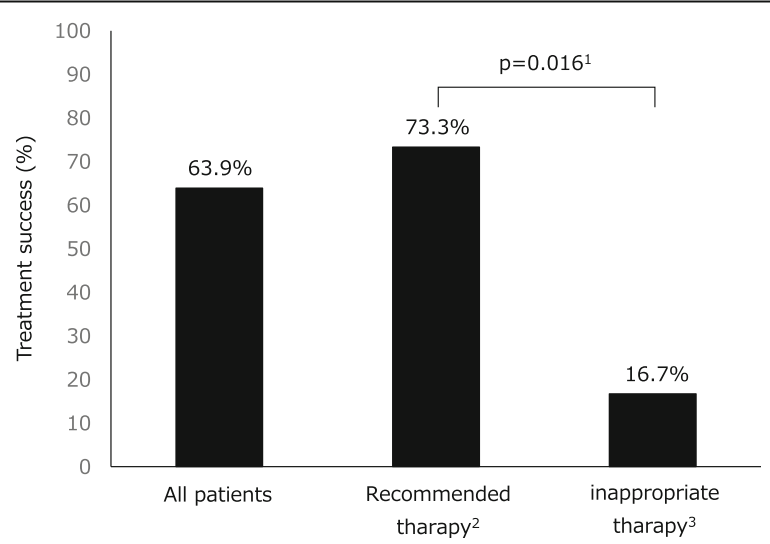

Fig. 1 Efficacy of Vancomycin tapered and pulsed-dose regimen in patients with recurrent Clostridioides difficile infection. This shows the treatment success rate of all patients under the vancomycin tapered and pulsed-dose regimen (VCM-TP); patients with VCM-TP according to the IDSA guideline (recommended therapy); and patients with VCM-TP departing from the recommended therapy (inappropriate therapy). ${ }^{1}$ Fisher Exact test, ${ }^{2}$ vancomycin tapered and pulsed-dose regimen according to IDSA guideline, ${ }^{3}$ vancomycin tapered and pulsed-dose regimen not following the IDSA guideline 
Table 2 Comparison of Clostridioides difficile infection patient characteristics in the treatment success and failure groups after the vancomycin tapered and pulsed-dose regimen

\begin{tabular}{|c|c|c|c|}
\hline & Treatment success $(n=23)$ & Treatment failure $(n=13)$ & $p$ value \\
\hline Male sex (\%) & $12(52.2)$ & $7(53.8)$ & $0.549^{2}$ \\
\hline Age, median (IQR $\left.{ }^{1}\right)$ & $80(73-83.5)$ & $83(76-87)$ & $0.281^{3}$ \\
\hline No. of CDI episodes, mode (range) & $3(2-7)$ & $3(2-7)$ & $0.115^{3}$ \\
\hline \multicolumn{4}{|l|}{ Underlying disease } \\
\hline Hypertension (\%) & $8(34.8)$ & $4(30.8)$ & $1.000^{2}$ \\
\hline Malignancy (\%) & $7(30.4)$ & $3(23.1)$ & $0.716^{2}$ \\
\hline Immunocompromised (\%) & $5(21.7)$ & $4(30.8)$ & $0.693^{2}$ \\
\hline Diabetes mellitus (\%) & $4(17.4)$ & $1(7.7)$ & $0.634^{2}$ \\
\hline Prior abdominal surgery (\%) & $3(13.0)$ & $2(15.4)$ & $1.000^{2}$ \\
\hline Chronic kidney disease (\%) & $3(13.0)$ & $1(7.7)$ & $1.000^{2}$ \\
\hline Chronic liver disease (\%) & $1(4.3)$ & $0(0)$ & $1.000^{2}$ \\
\hline Charlson comorbidity index, median (range) & $2(0-8)$ & $2(0-5)$ & - \\
\hline \multicolumn{4}{|l|}{ Drug use } \\
\hline Proton pump inhibitors (\%) & $10(43.5)$ & $6(46.2)$ & $0.877^{4}$ \\
\hline Histamine receptor-2 blockers (\%) & $3(8.3)$ & $0(0.0)$ & $0.288^{2}$ \\
\hline Probiotics (\%) & $19(82.6)$ & $12(92.3)$ & $0.634^{2}$ \\
\hline Antidiarrheals (\%) & $0(0.0)$ & $0(0.0)$ & - \\
\hline Antibiotic use prior 90 days (\%) & $21(91.3)$ & $12(92.3)$ & $1.000^{2}$ \\
\hline Penicillins & 7 & 1 & \\
\hline Cephalosporins & 6 & 4 & \\
\hline Carbapenems & 6 & 3 & \\
\hline Quinolones & 5 & 3 & \\
\hline Antitubercular & 6 & 5 & \\
\hline Others & 4 & 3 & \\
\hline Concomitant antibiotics during VCM-TP ${ }^{5}(\%)$ & $16(69.6)$ & $6(46.2)$ & $0.549^{2}$ \\
\hline \multicolumn{4}{|l|}{ Disease severity (\%) } \\
\hline Zar criteria [14] & & & $0.166^{4}$ \\
\hline$>2$ & $2(8.7)$ & $3(23.1)$ & \\
\hline$\leq 2$ & $21(91.3)$ & $10(76.9)$ & \\
\hline MN criteria [15] & & & $0.373^{4}$ \\
\hline mild & $6(16.7)$ & $7(53.8)$ & \\
\hline moderate & $14(60.9)$ & $5(38.5)$ & \\
\hline severe & $2(8.7)$ & $1(7.7)$ & \\
\hline Recommended VCM-TP ${ }^{6}(\%)$ & $22(95.7)$ & $8(61.5)$ & $0.016^{2}$ \\
\hline
\end{tabular}

${ }^{1} \mathrm{IQR}$ (interquartile range), ${ }^{2}$ Fisher Exact test, ${ }^{3}$ Mann-Whitney U test, ${ }^{4}$ Pearson $\mathrm{X} 2$ test, ${ }^{5} \mathrm{VCM}-\mathrm{TP}$ (vancomycin tapered and pulsed-dose regimen), ${ }^{6}$ vancomycin tapered and pulsed-dose regimen according to IDSA guideline

that inappropriate VCM-TP therapy, following unconventional guidelines, can increase the treatment failure rate (Fig. 1, Table 2). Mcfarland et al. hypothesized that the recommended VCM-TP allows gradual weeding out of $C$. difficile spores from the intestinal reservoir, resulting in low recurrence rate when pulsed doses of vancomycin are administered over an extended period (usually 3 weeks). Hence, we considered that inappropriate VCM-TP is more likely to result in treatment failure than recommended
VCM-TP because $C$. difficile spores are not completely removed due to interruptions in treatment.

Repetitive cycles of antibiotic-free periods and antibiotic pulses may be an effective strategy for treating recurrent CDI [11]. We consider that inappropriate therapy that violates the optimal schedule can lead to unnecessary VCM washout periods, thereby increasing the risk of regrowth and relapse of CDI. Accordingly, antimicrobial stewardship teams should implement the recommended VCM-TP. 
Tomas et al. reported that alterations to the indigenous microbiota responsible for colonization resistance to $C$. difficile and VRE persist during and after completion of tapering courses of vancomycin [16]. However, in our study, VRE was not detected in 20 patients in whom the VRE culture test was performed 41.9 days after treatment initiation. As our clinical data were limited, further studies in this regard are warranted.

There are certain limitations to this study. First, although our study was retrospective and observational, it only involved single-institutional data, and the sample size was inadequate. Additional prospective studies or clinical trials in multiple centers with larger numbers of patients are warranted. Second, it was difficult to confirm whether recurrent CDI was caused by the same C. difficile strain. The recurrence rate of CDI by the same bacterial strain is $45.8 \%-83.0 \%[17,18]$. Furthermore, if recurrent CDI was caused by the same bacterial strain, determining the specific cause is difficult considering the environmental factors or colonization in the intestinal tract.

\section{Conclusions}

Our findings suggest that VCM-TP can be a good therapeutic option for recurrent CDI in Japanese settings. Administration of the recommended VCM-TP is important to ensure a high rate of treatment success. Hence, antimicrobial stewardship teams should support the implementation of recommended VCM-TPs.

\section{Abbreviations}

CDI: Clostridioides (Clostridium) difficile infection; Cl: Confidence interval; IQR: Interquartile range.; OR: Odds ratio; VCM-TP: Vancomycin tapered and pulsed-dose regimen; VRE: Vancomycin-resistant enterococci

\section{Acknowledgments}

We thank all clinicians of the antimicrobial stewardship team at the Tosei General Hospital.

\section{Authors' contributions}

TU and $\mathrm{AO}$ designed the research. TU and $\mathrm{AO}$ analyzed the data. TU, Al, MY, $\mathrm{TM}, \mathrm{KK}$, and $\mathrm{YI}$ interpreted the results. TU was a major contributor in drafting the manuscript. All authors read and approved the final version of the manuscript.

\section{Funding}

There are no funding sources for this study.

\section{Availability of data and materials}

All data generated or analyzed in this study are included in this published article.

\section{Ethics approval and consent to participate}

This study was approved by the ethics committee of the Tosei General Hospital (receipt no. 769) and was performed according to the Declaration of Helsinki. The identities of the study subjects were kept confidential during data collection.

\section{Consent for publication}

Not applicable.

\section{Competing interests}

The authors declare that they have no competing interests.

\section{Author details}

'Department of Pharmacy, Tosei General Hospital, 160, Nishi oiwakecho, Seto, Aichi 489-8642, Japan. ${ }^{2}$ Department of Infection and Prevention, Tosei General Hospital, 160, Nishi oiwakecho, Seto, Aichi 489-8642, Japan. ${ }^{3}$ Department of Infectious Disease, Tosei General Hospital, 160, Nishi oiwakecho, Seto, Aichi 489-8642, Japan. ${ }^{4}$ College of Pharmacy, Kinjo Gakuin University, 2-1723, Omori, Moriyama-ku, Nagoya, Aichi 463-8521, Japan.

Received: 14 May 2019 Accepted: 8 July 2019

Published online: 08 August 2019

\section{References}

1. Thongprayoon C, Cheungpasitporn W, Phatharacharukul P, Mahaparn P, Bruminhent J. High mortality risk in chronic kidney disease and end stage kidney disease patients with Clostridium difficile infection: a systematic review and meta-analysis. J Nat Sci. 2015;1:e85.

2. Centers for Disease Control and Prevention. Nearly half a million Americans suffered from Clostridium difficile infections in a single year. 2015. https:// www.cdc.gov/media/releases/2015/p0225-clostridium-difficile.html. Accessed 21 Mar 2019

3. Yasunaga $\mathrm{H}$, Horiguchi $\mathrm{H}$, Hashimoto $\mathrm{H}$, Matsuda S, Fushimi K. The burden of Clostridium difficile-associated disease following digestive tract surgery in Japan. J Hosp Infect. 2012;82:175-80.

4. Fukuda $H$, Yano $T$, Shimono N. Inpatient expenditures attributable to hospital-onset Clostridium difficile infection: a nationwide case-control study in Japan. Pharmacoeconomics. 2018;36:1367-76.

5. Abou Chakra CN, Pepin J, Sirard S, Valiquette L. Risk factors for recurrence, complications and mortality in Clostridium difficile infection: a systematic review. PLoS One. 2014;9:e98400.

6. Reveles KR, Mortensen EM, Koeller JM, Lawson KA, Pugh MJV, Rumbellow $S A$, et al. Derivation and validation of a Clostridium difficile infection recurrence prediction rule in a national cohort of veterans. Pharmacotherapy. 2018;38:349-56.

7. Pepin J, Routhier S, Gagnon S, Brazeau I. Management and outcomes of a first recurrence of Clostridium difficile-associated disease in Quebec. Canada Clin Infect Dis. 2006:42:758-64.

8. Kunishima $\mathrm{H}$, Ito K, Laurent T, Abe M. Healthcare burden of recurrent Clostridioides difficile infection in Japan: a retrospective database study. J Infect Chemother. 2018;24:892-901.

9. McDonald LC, Gerding DN, Johnson S, Bakken JS, Carroll KC, Coffin SE, et al. Clinical practice guidelines for Clostridium difficile infection in adults and children: 2017 update by the Infectious Diseases Society of America (IDSA) and Society for Healthcare Epidemiology of America (SHEA). Clin Infect Dis. 2018;66:987-94.

10. The Japanese Society of Chemotherapy and The Japanese Association for Infectious Diseases. The Japanese clinical practice guidelines for management of Clostridioides (Clostridium) difficile infections. Tokyo: Kyorinsha; 2018.

11. McFarland LV, Elmer GW, Surawicz CM. Breaking the cycle: treatment strategies for 163 cases of recurrent Clostridium difficile disease. Am J Gastroenterol. 2002;97:1769-75.

12. Majors D, Ellis P. Risk factors for recurrent Clostridium difficile infections and strategies to decrease readmissions in a community hospital. Hosp Pharm. 2015;50:1003-10.

13. Sirbu BD, Soriano MM, Manzo C, Lum J, Gerding DN, Johnson S. Vancomycin taper and pulse regimen with careful follow-up for patients with recurrent Clostridium difficile infection. Clin Infect Dis. 2017;65:1396-9.

14. Zar FA, Bakkanagari SR, Moorthi KM, Davis MB. A comparison of vancomycin and metronidazole for the treatment of Clostridium difficile-associated diarrhea, stratified by disease severity. Clin Infect Dis. 2007;45:302-7.

15. Yamagishi Y, Hagihara M, Hiroshige M. Evaluation of newly developed Clostridium difficile infection severity scoring system "MN criteria" for Clostridium difficile infection patients in Japan. Journal of Japanese Association for Anaerobic Infection Research. 2017:47:66-75.

16. Tomas ME, Mana TSC, Wilson BM, Nerandzic MM, Joussef-Piña S, QuiñonesMateu ME, et al. Tapering courses of oral vancomycin induce persistent disruption of the microbiota that provide colonization resistance to 
Clostridium difficile and vancomycin-resistant enterococci in mice. Antimicrob Agents Chemother. 2018;62:e02237-17.

17. Figueroa I, Johnson S, Sambol SP, Goldstein EJ, Citron DM, Gerding DN. Relapse versus reinfection: recurrent Clostridium difficile infection following treatment with fidaxomicin or vancomycin. Clin Infect Dis. 2012;55(Suppl 2):S104-9.

18. Durovic A, Widmer AF, Frei R, Tschudin-Sutter S. Distinguishing Clostridium difficile recurrence from reinfection: independent validation of current recommendations. Infect Control Hosp Epidemiol. 2017;38:891-6.

\section{Publisher's Note}

Springer Nature remains neutral with regard to jurisdictional claims in published maps and institutional affiliations.

Ready to submit your research? Choose BMC and benefit from:

- fast, convenient online submission

- thorough peer review by experienced researchers in your field

- rapid publication on acceptance

- support for research data, including large and complex data types

- gold Open Access which fosters wider collaboration and increased citations

- maximum visibility for your research: over $100 \mathrm{M}$ website views per year

At $B M C$, research is always in progress.

Learn more biomedcentral.com/submissions 